\title{
SINGLE AUTHOR PAPERS IN MASHHAD UNIVERSITY OF MEDICAL SCIENCES (MUMS) IN THE RECENT 10 YEARS
}

\author{
Faeze Movahedianfar ${ }^{1}$, Hadi Ghasemi², Saeed Eslami Hasan Abadi ${ }^{2}$
}

1: Student Research Committee, Faculty of Medicine, Mashhad University of Medical Sciences, Mashhad, Iran

2: Department of bioinformatics, Faculty of Medicine, Mashhad University of Medical Sciences, Mashhad, Iran

Correspondence:

Saeed Eslami Hasan Abadi, Tel: +989154403990, E-mail: EslamiS@mums.ac.ir

\section{TYPE OF ARTICLE: CONFERENCE ABSTRACT}

\begin{abstract}
Introduction: Bibliometric analysis can explore the patterns of authorship. The single authorship is an unpleasant phenomenon in research, and we investigate the single author papers' trend in Mashhad University of Medical sciences (MUMS) in the recent 10 years.

Methods: We searched PubMed for articles that have Mashhad University of Medical sciences (MUMS) in affiliations and filtered data by date (2006-2015). Bibliometric graphs were drawn by VOSViewer software. Graphs showed the effect of special authors in all data.

Results: Quantitative data analysis explore that the average of authors in each article has increased (3.32 in 2006 and 5.36 in 2015): $2.6 \%$ of 939 articles and 3.1\% of 868 articles (in 2015 and 2016 respectively) were single author papers; $68 \%$ of single author papers were journal articles, and the rest were other types of articles such as editorials, comments, letters, and case reports.

Conclusion: Bibliometric studies show that single authorship was declining. This paper may be considered as a baseline study for the bibliometric information. Future studies can compare the single authorship between MUMS and other universities and monitor the trend of single authorship.

KEYWORDS: Single authorship, Bibliometric indicators, Mashhad university of medical sciences
\end{abstract}

